\title{
Efficacy of methotrexate as anti-inflammatory and anti-proliferative drug in dermatology: Three case reports
}

\author{
ROXANA-IOANA NEDELCU ${ }^{1,2}$, MIHAELA BALABAN ${ }^{1,2}$, GABRIELA TURCU $^{1-3}$, \\ ALICE BRINZEA $^{1,3,4}$, DANIELA ADRIANA ION ${ }^{1}$, MIHAELA ANTOHE ${ }^{1,2}$, ANASTASIA HODOROGEA ${ }^{1,4}$, \\ ANDREEA CALINESCU ${ }^{1,4}$, ANCA IOANA BADARAU ${ }^{1}$, CRISTIANA GABRIELA POPP ${ }^{4}$, MIRELA CIOPLEA ${ }^{4}$, \\ LUCIANA NICHITA $^{4}$, SILVIA POPESCU ${ }^{1}$, CARMEN DIACONU ${ }^{5}$, CORALIA BLEOTU ${ }^{5}$, DANIEL PIRICI ${ }^{6}$, \\ RALUCA POPESCU ${ }^{1,4}$, CATALIN MIHAI POPESCU ${ }^{1,4}$ and SABINA ANDRADA ZURAC ${ }^{1,4}$ \\ ${ }^{1}$ Faculty of Medicine, 'Carol Davila' University of Medicine and Pharmacy, 050474 Bucharest; ${ }^{2}$ Department of \\ Dermatology, Derma $360^{\circ}$ Clinic, 011273 Bucharest; ${ }^{3}$ Department of Ambulatory, National Institute for \\ Infectious Diseases 'Prof. Dr. Matei Balș', 020105 Bucharest; ${ }^{4}$ Department of Dermatology, Colentina Clinical Hospital, \\ 020125 Bucharest; ${ }^{5}$ Department of Cellular and Molecular Pathology, 'Stefan S. Nicolau' Institute of Virology, \\ 030304 Bucharest; ${ }^{6}$ Department of Pathology, University of Medicine and Pharmacy of Craiova, 200349 Craiova, Romania
}

Received July 31, 2018; Accepted October 17, 2018

DOI: $10.3892 / \mathrm{etm} .2019 .7511$

\begin{abstract}
Methotrexate (MTX) is a folic acid analog with anti-proliferative (anti-neoplastic, cytotoxic), immunosuppressive and anti-inflammatory properties, which has been used in the treatment of various cutaneous disorders, such as psoriasis, keratoacanthoma, pityriasis rubra pilaris, atopic dermatitis, mycosis fungoides, bullous skin diseases, systemic sclerosis, morphea, lupus erythematosus, dermatomyositis and crusted scabies. Inhibition of cell proliferation is explained through its role in blocking DNA/RNA synthesis, by inhibiting dihydrofolate reductase, necessary for the production of pyrimidine and purine nucleotides. An anticancer effect can be related to $\alpha$-oxoaldehyde metabolism (MTX increases methylglyoxal levels). Its anti-inflammatory property is based on the inhibition of 5-aminoimidazole-4-carboxamide ribonucleotide transformylase, thus increasing intracellular and extracellular adenosine, a purine nucleoside with anti-inflammatory effect. This drug can limit inflammation by scavenging free radicals and decreasing malondialdehyde-acetaldehyde protein-adduct production. Moreover, the anti-proliferative and anti-inflammatory effects can also be related to inhibition of the DNA methylation pathway, thus inhibiting methionine formation. The aim of the present study was to report various dermatological cases from our daily practice that
\end{abstract}

Correspondence to: Dr Mihaela Balaban, Department of Dermatology, Derma $360^{\circ}$ Clinic, 3A Comana Street, 011273 Bucharest, Romania

E-mail: mihaela.cernat@gmail.com

Key words: methotrexate, antiproliferative, immunosuppressive, anti-inflammatory, psoriasis, keratoachantoma demonstrate the efficacy of MTX in the treatment of cutaneous diseases, highlighting different mechanisms of action: its anti-inflammatory effect in psoriasis and its anti-proliferative, and anti-neoplastic effect in well-differentiated squamous cell carcinoma or in keratoacanthoma. Moreover, different administration pathways and doses are addressed. Assessment of the treatment plan, clinical improvement of cutaneous lesions, biologic evaluation, final aesthetic result, quality of life, as well as potential adverse effects and drug tolerance related to each case mentioned.

\section{Introduction}

Methotrexate (MTX) (amethopterin or 4-amino-N10-methyl pteroylglutamic acid) is a folic acid analog, whose effects can be classified into anti-proliferative [dihydrofolate reductase (DHFR)-mediated] and anti-inflammatory effects (non-DHFR-mediated) (1).

In the center of the anti-inflammatory pathway is a purine nucleoside known as adenosine, which has the capacity to fight against the inflammatory process (2). The antiproliferative, antineoplastic, and cytotoxic effects are based on decreased nucleic acid formation in activated $\mathrm{T}$ cells and in keratinocytes (3).

The aim of the study was to synthesize the most relevant information regarding the mechanism of action of MTX in dermatological pathology, demonstrating each of them with representative clinical cases.

MTX and MTX polyglutamates (MTXPGs) molecules have the ability to inhibit a folate-dependent enzyme, involved in purine nucleotides synthesis, termed 5-aminoimidazole-4-carboxamide ribonucleotide (AICAR) transformylase (1,2,4-6).

This enzyme is involved in the transformation of AICAR in formyl-AICAR, a purinic DNA precursor. Thus, in the absence of the function of this transformylase, AICAR accumulates within the cell, which results in the inhibition of 
adenosine deaminase, leading to elevated levels of adenosine in the extracellular space $(1,2,4,5)$.

Adenosine is the key molecule regarding the anti-inflammatory response of MTX (2). The anti-inflammatory effect is the result of the interaction of adenosine with adenosine receptors on the cell surface, a mechanism that inhibits leukocyte chemotaxis, oxidative inflammation in neutrophils/monocytes and cytokine synthesis from monocyte/macrophages (TNF- $\alpha$, IL-6,-8,-10 and -12) (1,2,4,5). Moreover, IL-1, IL-4, IL-13 and INF- $\gamma$ release is decreased $(1,2,5)$. Adenosine receptors, also called $\mathrm{P} 1$ receptors, can be divided into A1, A2a, A2b and A3 (2). The A2a receptor is associated with the greatest anti-inflammatory effect $(2,7)$. MTX promotes apoptosis in activated $\mathrm{CD}^{+} \mathrm{T}$ lymphocyte and reduces neovascularization (5). The combination of malondialdehyde (MDA) and acetaldehyde (AA) can lead to malondialdehyde-acetaldehyde (MAA)-protein-adduct, markers of oxidative stress. It was previously shown that, by decreasing the production of these compounds and by scavenging free radicals, MTX could have an additional anti-inflammatory effect (8-12). The anti-inflammatory effect of MTX was demonstrated in diseases such as psoriasis (moderate to severe en plaque lesions, psoriatic arthritis, erythrodermic and pustular forms), bullous diseases, vasculitis, atopic dermatitis, lupus erythematosus, rheumatoid arthritis and sclerodermia $(3,5)$.

\section{Case reports}

Case 1: Chronic plaque psoriasis vulgaris. A 57-year-old male patient presented for a disseminated eruption involving the trunk and the limbs, including the elbows and the knees, which was evolving for a few months. The patient was diagnosed with psoriasis vulgaris for more than 30 years, with lesions affecting a small body surface (mainly elbows and knees), for which he was treated with topical therapies (vitamin D analogues, topical corticosteroids and emollients). The evolution of the disease was chronic, with remissions and relapses until a few months before, when the lesions became more disseminated and severe.

Clinical examination revealed multiple erythematous, well-demarcated large plaques, with a thick ivory-white scale covering the lesions, mildly pruritic. Auspitz sign and wax candle sign were present as well. The scalp and the genitalia were spared. The nails of the hands had typical psoriatic signs, such as thickening of the nail plate, distal onycholysis and a yellow colour (oil spot sign). The patient did not have any arthralgia or joint swelling and he was otherwise healthy. Evaluation of the lesions concluded with a Psoriasis Area and Severity Index (PASI) of 32.4 points.

Blood test evaluation (hemogram parameters, transaminases, serum urea and creatinine, total and direct bilirubin, alkaline phosphatase) was within normal values. The tests for hepatitis B/C and HIV were negative. The patient was prescribed oral MTX of $15 \mathrm{mg} /$ week in a single dose, concomitant with folic acid administration (5 $\mathrm{mg} / \mathrm{day}$, excepting the day of administration of MTX).

During the treatment, the hemogram, the hepatic and renal tests had normal values, including those made just after the beginning of the therapy, with a good tolerance of the drug (only a mild nausea). The drug was well-tolerated (only a mild nausea) and a gradual improvement with regard to the cutaneous lesions was observed. After 5 months of treatment, clinical evaluation revealed a PASI of 5.0 points. The aspect of the nails remained stable, without significant benefit. Although the renal and hepatic functions were unaffected by the therapy, the medium corpuscular volume (MCV) had an abnormally lower value than it had been before. Taking into consideration the PASI 75 improvement, MTX administration was interrupted, in order to prevent any possible adverse events, related to bone marrow toxicity. The patient was prescribed a topical combination of calcipotriol/ betamethasone gel for 1 month, resulting in a PASI reduction until 0.7 points.

Administration and pharmacokinetics of MTX in psoriasis vulgaris. The route of administration can be oral, intramuscular or subcutaneous, and on a weekly basis. The i.v. route is also available for some products (MTX $100 \mathrm{mg} / \mathrm{ml}$; Hospira UK Ltd., SmPC, Maidenhead, UK), whereas other products are only for s.c. use (Nordimet SmPC, Berkshire, UK).

In case of oral intake of the drug, the possibility of dividing the dose into three equal parts taken every $12 \mathrm{~h}$ during a 24-h period, may help to reduce digestive adverse events (3). The parenteral administration can also help the patient regarding digestive tolerance (4).

After administration, the highest serum concentration is achieved in 1-2 $\mathrm{h}$, being faster for the the intramuscular pathway $(<1 \mathrm{~h})(3)$.

In the blood, the drug circulates into two forms (MTX and its active form 7-OH MTX), bound to albumin at a percentage of $50-70 \%(4,5)$. The serum half-life of the drug is 6-7 $\mathrm{h}$, but longer for MTXPGs, which are the long-acting active metabolites and which can last several months $(4,5,13)$. Moreover, within $24 \mathrm{~h}$ after administration, up to $80 \%$ of the amount of the molecule is eliminated unchanged through renal system $(4,5)$. Patients taking salycilates, sulphonamides, tetracycline, chloramphenicol and other drugs, are at great risk of toxicity, considering the fact that albumin binding of MTX is reduced (3). Moreover, other therapies such as ciclosporin, and NSAIDs, reduce kidney excretion of MTX and may increase its levels in the body and the risk of side effects (3). For the majority of cutaneous diseases treated using MTX, the usual dose can be between 10 and $25 \mathrm{mg} /$ week, with an average dose of $15 \mathrm{mg} /$ week (5). MTX therapy needs several weeks until its efficacy is proved, considering the fact that it has a slow action (3).

Adverse effects of MTX and management regarding the therapy in psoriasis vulgaris

Hepatotoxicity. Hepatic toxicity refers to abnormal transaminases, hepatic fibrosis and cirrhosis. The fibrosis can be reversible in case of drug intake cessation $(5,14)$. Patients at risk are heavy drinkers, those with abnormal transaminases, with hepatic disease, hepatitis $\mathrm{B}$ and $\mathrm{C}$, those taking other hepatotoxic therapies, obese or are diabetic $(5,15)$. Pretreatment assessment is based on transaminases, alkaline phosphatase, bilirubin and albumin, as well as serologic tests for hepatitis B and C. Hepatic test have to be repeated every month in the first 6 months and then every 1-2 months (5).

Hepatotoxicity is dose-related; however, there is controversy about hepatic biopsy. Subsequently, if hepatic risk 
factors are absent, a hepatic biopsy is recommended after a cumulative dose of 3.5-4 g of MTX $(16,17)$. Moreover, the $\mathrm{SmPC}$ for Nordimet (approved by the European Medicines Agency) states that there is no evidence to support the idea of performing a liver biopsy in evaluating liver toxicity in rheumatological patients treated with MTX (18). This drug cannot be administered if the patient is alcoholic, if hepatic function tests reveal anomalies and in case of hepatitis B and C, diabetes mellitus, obesity, or simultaneous intake of other drugs, which can affect the liver.

At present, periodic evaluation of serum procollagen type III N-terminal peptide (PIINP) in countries where it is available and considering the fact that elevated levels can be associated with fibrotic liver damage, are useful in avoiding invasive liver biopsies (3). Moreover, SmPC for Nordimet states that 'further research is needed to establish whether serial liver chemistry tests or propeptide of type III collagen can detect hepatotoxicity sufficiently' (18).

Haematologic toxicity. Bone marrow suppression induced by MTX can result in leukocytopenia, trombocytopenia, pancytopenia and megaloblastic anemia (4).

Patients that are more prone to developing hematologic adverse events are those with renal impairment, a decreased level of serum albumin, or those taking drugs that interact with MTX, age above 65 years and patients suffering from other systemic conditions and infections $(5,19)$. Pretreatment evaluation of a patient includes complete blood cell count, which is repeated after the first week of therapy, followed by measurements every 2 weeks for the next 2 months, and then every 2-3 months $(3,5)$. An HIV test is mandatory.

The drug cannot be administered in case of blood test anomalies (leukocytes $<3,500 / \mathrm{mm}^{3}$, platelets $<100,000 / \mathrm{mm}^{3}$ ), or immunodeficiency syndromes (HIV) (5). Elevation of the value of MCV is a reliable sign of bone marrow toxicity and requires a supplementation of acid folic dosage and a decreased MTX dosage (4). Moreover, mucositis is a cutaneous disorder that predicts the risk of pancytopenia, which can be prevented by folic acid supplementation (4,5). Other suggestive signs of pancytopenia are cough, breathing difficulty, bleeding, fever, nausea, and cyanosis (3). Myelosuppression usually occurs in case of inadequate dose intake (daily dose instead of weekly); thus, proper information regarding weekly administration of MTX should be given to the patient (5).

Gastro-intestinal toxicity. Patients may experience nausea, vomiting, less often diarrhea and mucositis (5). Measures, such as dose-splitting, concomitant folic acid administration or other route than orally can help curtail digestive intolerance $(4,20)$. The drug cannot be taken by individuals with acute peptic ulcer (3).

Importance of renal function. Considering that MTX is excreted through kidney, an abnormal renal function may result in high levels of MTX and thus, several adverse effects. Baseline evaluation prior to treatment should include blood urea nitrogen, serum creatinine, urinalysis and determination of creatinine clearance (24-h urine or using Cockrogt-Gault equation) (5). Patients with severe renal insufficiency (Glomerular Filtration Rate $<10 \mathrm{ml} / \mathrm{min}$ ) or those on dialysis cannot take MTX (5). A GFR $>10 \mathrm{ml} / \mathrm{min}$ requires a dose reduction (5). The recommendation is to repeat the renal function tests (BUN, creatinine) every 2 months (5).
Other possible adverse effects. A patient taking MTX can also experience fatigue, headaches, dizziness, nausea, malaise, anorexia, alopecia, or accentuation of sun-induced redness $(4,5)$. Pulmonary side effects are represented by pulmonary fibrosis and acute pneumonitis (5). The drug is contraindicated in pregnant women or during breastfeeding, because of the potential teratogenicity, respectively, the risk of secretion in the breast milk. The drug can induce spontaneous abortion during the first trimester of pregnancy $(5,21)$.

Men can be affected by reversible oligospermia and possible genetic anomaly of the fetus may also occur $(5,21)$. Pretreatment evaluation includes pregnancy test, which should be periodically repeated. Patients are advised to wait one ovulatory cycle (in the case of a woman) or 3 months (if the patient is a man) until conception $(5,21)$.

Other important aspects regarding the treatment. Folic and folinic acid supplementation during the treatment with MTX has been controversial, with claims that it may reduce the efficacy of MTX $(22,23)$, as well as claims that it is beneficial (24). A Cochrane review concluded that there is some safety advantage in using folic or folinic supplementation (25). It can decrease the risk of myelosuppression, the risk of stomatitis, hepatic toxicity and enhances gastro-intestinal tolerance. There are several approaches regarding the administration regimen of folic acid, but usually the dose ranges between 1 and $5 \mathrm{mg}$, with the possibility of increasing the dose of folic acid in case of severe MTX-induced toxicity (5).

In case of any overdose of MTX, the drug of choice is folinic acid, a reduced folic compound, whose action is independent of DHFR and which does not need activation (3). A Cochrane review published in 2014 concluded in the abstract that 'It does not appear that supplementation with either folic or folinic acid has a statistically significant effect on the efficacy of MTX in treating RA (as measured by RA disease activity parameters such as tender and swollen joint counts, or physician's global assessment scores)' (25). Other treatment options are also available for psoriasis $(26,27)$.

\section{The antiproliferative effects}

General information. As a folic acid analog, the molecule of the drug suffers similar transformations as folates. MTX is a prodrug and it needs the reduced folate carrier to facilitate its penetration into the cell, the place where it is activated in MTXPGs through the action of folylpolyglutamate synthetase, by adding glutamate groups $(1,3,28)$. MTXPGs are long-acting metabolites that persist for months, which explains the prolonged effect and the need for only weekly administration $(2,4)$. The substance is characterized with affinity to fibroblast, red blood cells, myeloid cells, and liver cells $(5,13)$.

MTX and MTXPGs derivatives inhibit cellular replication by inhibiting folate acid-dependent enzymes. Those involved in the pyrimidine production are DHFR and thymidylate synthetase, whereas those related to purine synthesis are AICAR transformylase and glycinamide ribonucleotide (GAR) transformylase. Moreover, DNA methylation is affected by the inhibition of methionine synthase, an enzyme involved in the transformation of homocysteine in methionine $(4,5)$

DHFR is an important enzyme involved in the activation of folate compounds, more specifically in the reduction of DHFR in THF. DNA and RNA synthesis is based on purine 


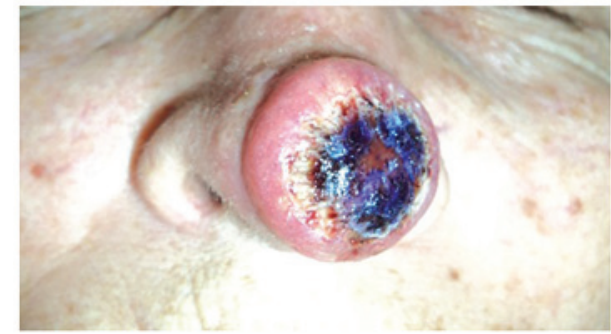

Figure 1. A 72-year-old female patient presented with a nodule located on the tip of the nose, whose clinical appearance was suggestive of keratoacanthoma.

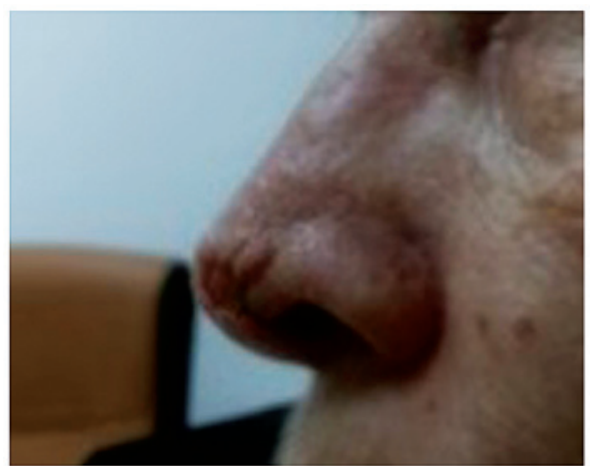

Figure 2. After receiving 3 injections complete remission was achieved after the third injection. At the end of the therapy, only a minor defect was present on the nose.

and pyrimidine nucleotide formation, which in turn requires the presence of THF to act as a co-factor for many enzymatic reactions $(1,4,5)$.

Moreover, DNA synthesis can be impaired through the reduction of thymidylic acid synthesis, through inhibition of thymidylate synthetase. This pathway does not affect ARN synthesis, because thymine is a pyrimidine nitrogenous base found only in the DNA structure. The drug is characterized by a specificity regarding the cell cycle, as it is active only in the S-phase $(4,5,13)$.

An anticancer effect, as well as toxic effects, can be associated with $\alpha$-oxoaldehyde metabolism. Thus, MTX increases the methylglyoxal level, which contributes to the glycation of biomolecules, a pathway associated with antineoplastic results $(1,8)$. Moreover, increasing oxidative stress within the cell leads to MTX promoting apoptosis and inhibiting proliferation $(1,29)$.

Thus, the antiproliferative, cytotoxic effect of MTX was demonstrated in the treatment of keratoacanthoma.

Case 2: Keratoacanthoma of the nose. A 72-year-old female patient was referred for a nodule located on the tip of the nose, rapidly evolving over the past 3 weeks and whose clinical appearance was suggestive of keratoacanthoma (Fig. 1). The patient admitted having a similar lesion on the nose one year before, which reappeared three times and which was treated each time using electrocauterization. Clinical examination revealed an erythematous, well-demarcated nodule, $2.5 \mathrm{~cm}$ in diameter, with a crateriform hyperkeratotic core.

The patients received 3 injections with intralesional MTX every 2 weeks, with a total dose of $60 \mathrm{mg}$. The tumor was

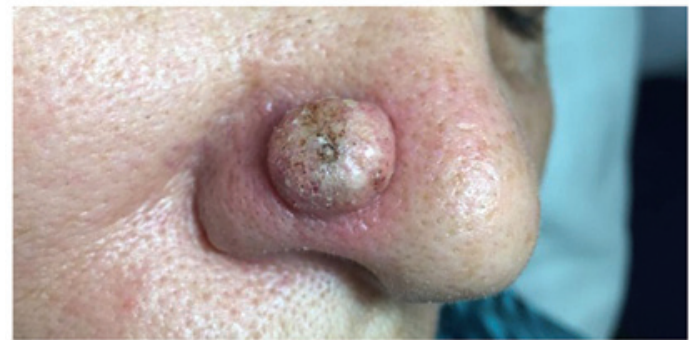

Figure 3. A 69-year-old female patient presented with a red dome-shaped nodule, $1.5 \mathrm{~cm}$ in diameter, near the alar crease of the nose, which was consistent with the diagnosis of keratoacanthoma.

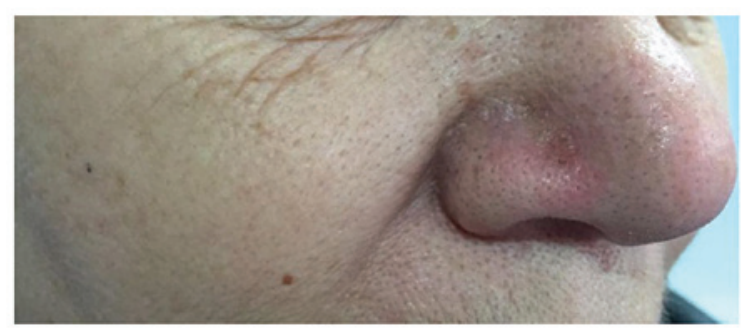

Figure 4. The therapeutic approach was based on only one injection with intralesional MTX. After 2 weeks, the nodule began to shrink and to develop a central necrosis, followed by spontaneous rupture from the base and a complete remission.

injected in four quadrants with $2 \mathrm{ml}$ of MTX (a concentration of $10 \mathrm{mg} / \mathrm{ml}$ ) until blanching was achieved. During the treatment, a progressive regression of the lesion was evident. A complete remission was obtained after the third injection, with a good tolerance of the drug and without adverse effects. At the end of the therapy, only a minor defect was present on the nose (Fig. 2); thus, the patient was sent to the Department of Plastic Surgery, in order to evaluate the esthetic result, the oncologic safety of the lesion and histopathological evaluation.

Case 3: Keratoacanthoma of the nose. A 69-year-old female patient presented with a red dome-shaped nodule, $1.5 \mathrm{~cm}$ in diameter, near the alar crease of the nose, with a fast evolution within 2 months (Fig. 3). Clinical examination was consistent with the diagnosis of keratoacanthoma.

The therapeutic approach was based on only one injection with intralesional MTX $(1 \mathrm{ml}$ of a $12.5 \mathrm{mg} / \mathrm{ml})$ injected in 4 quadrants and at the base of the tumor, until a whitish color was observed. After 2 weeks, the nodule began to shrink and to develop a central necrosis, followed by spontaneous rupture from the base and a complete remission (Fig. 4). The patient did not experience any adverse effect during the therapy and no recurrences were evident within the first year of follow-up.

\section{Discussion}

Administration in keratoacanthoma. Keratoacanthoma is a cutaneous disorder affecting mainly the sun-exposed parts of the body of older patients, clinically presenting as an erythematous round nodule with sharp demarcation, which has a typical crateriform keratotic core. In the literature, this tumor is considered either a sub-type of well-differentiated 
squamous cell carcinoma, or a squamous lesion that can spontaneous involute. Even though complete surgical excision is the treatment of choice, intralesional MTX has proven its benefits in various cases of large keratoacanthoma of the face, significantly reducing the tumor size before surgical excision, with superior aesthetic results $(30,31)$.

The therapy of keratoacanthoma with intralesional administration of MTX is an 'off-label' indication, being based on a number of cases reported in the literature. The dose is $1 \mathrm{ml}$ of solution with different concentrations, depending on tumor dimension $(5,12.5$ and $25 \mathrm{mg} / \mathrm{mg}$ ) injected into four quadrants, until blanching. The reduction depends on every single case, but usually 1-4 injections are needed in order to achieve an optimal result. There is no fixed recommendation regarding the interval between doses; thus, the sessions can be repeated every 7-49 days (an average of 2-3 weeks). The complete response rate was $92 \%$ based on a series of 38 cases (more than half from the literature), but it might be influenced to a certain extent by publication bias $(30,31)$.

Adverse effects of MTX in keratoacanthoma. Even though there is no consensus regarding the evaluation before intralesional therapy as the one in psoriasis vulgaris, it is recommended to exclude those patients in whom the treatment might be contraindicated. Usually, the intralesional therapy is well-tolerated, without significant adverse effects, compared to those encountered in the systemic administration of MTX. Nevertheless, pancytopenia occurred in 2 patients who had severe renal impairment $(32,33)$

The immunosupresive effect. MTX effects are related to inhibition of two types of immune responses: humoral immune response (antibody-mediated) and cellular immune response (lymphocyte mediated), by reducing lymphocites migration in the skin, and modulating intra- and intercelluar signaling. By decreasing the level of cytokines such as TNF- $\alpha$, IL-10, IL-12, it can be effective in several immune-mediated skin disorders (4).

This study highlights the various mechanisms of action of MTX in different skin diseases. We explain both the therapeutic and possible side effects of MTX in intralesional and non-intralesional routes of administration. In our opinion, illustrating with clinical cases from our professional experience may be explanatory for the intricate pathways through which MTX exerts its anti-inflammatory and anti-proliferative actions.

\section{Acknowledgements}

Not applicable.

\section{Funding}

This study was partially supported by a grant from the Ministry of Research and Innovation, CNCS-UEFISCDI (project no. PN-III-P4-ID-PCE-2016-0641) within PNCDI-III; and from the Romanian Ministry of Research and Innovation, CCCDI-UEFISCDI (project no. 61PCCDI/2018 PN-III-P1-1.2-PCCDI-2017-0341) within PNCDI-III.

\section{Availability of data and materials}

All data generated or analyzed during this study are included in the published article.

\section{Authors' contributions}

RIN, MB, GT, AB, MA, AH, AC, RP, CMP were contributed to the conception of the study, acquisition, analysis, interpretation of data, and drafting the manuscript. DAI, AIB, CGP, MC, $\mathrm{LN}, \mathrm{SP}, \mathrm{CD}, \mathrm{CB}, \mathrm{DP}, \mathrm{SAZ}$ were responsible for the conception and design of the study, and revising it critically for important intellectual content. All authors approved the final version to be published and agreed to be accountable for all aspects of the study in ensuring that questions related to the accuracy or integrity of any part of the study are appropriately investigated and resolved.

\section{Ethics approval and consent to participate}

Not applicable.

\section{Patient consent for publication}

Patient consent for the images was obtained from patients included in the sudy.

\section{Competing interests}

The authors declare that they have no competing interests.

\section{References}

1. Sramek M, Neradil J and Veselska R: Much more than you expected: the non-DHFR-mediated effects of methotrexate. Biochim Biophys Acta Gen Subj 1861: 499-503, 2017.

2. Chan ESL and Cronstein BN: Molecular action of methotrexate in inflammatory diseases. Arthritis Res 4: 266-273, 2002.

3. Pathirana D, Ormerod AD, Saiag P, Smith C, Spuls PI, Nast A, Barker J, Bos JD, Burmester GR, Chimenti S, et al: European S3-guidelines on the systemic treatment of psoriasis vulgaris. J Eur Acad Dermatol Venereol 23: 1-70, 2009.

4. Belgi G and Friedmann PS: Traditional therapies: glucocorticoids, azathioprine, methotrexate, hydroxyurea. Clin Exp Dermatol 27: 546-554, 2002.

5. Bangert CA and Costner MI: Methotrexate in dermatology. Dermatol Ther 20: 216-228, 2007.

6. Negrei C, Ginghina O, Caruntu C, Burcea-Dragomiroiu GTA, Jinescu $G$ and Boda D: Investigation relevance of methotrexate polyglutamates in biological systems by high performance liquid chromatography. Rev Chim 66: 766-768, 2015.

7. Strober BE and Menon K: Folate supplementation during methotrexate therapy for patients with psoriasis. J Am Acad Dermatol 53: 652-659, 2005.

8. Zimmerman MC, Clemens DL, Duryee MJ, Sarmiento C, Chiou A, Hunter CD, Tian J, Klassen LW, O'Dell JR, Thiele GM, et al: Direct antioxidant properties of methotrexate: inhibition of malondialdehyde-acetaldehyde-protein adduct formation and superoxide scavenging. Redox Biol 13: 588-593, 2017.

9. Negrei C, Caruntu C, Ginghina O, Burcea-Dragomiroiu GTA, Toderescu CD and Boda D: Qualitative and quantitative determination of methotrexate polyglutamates in erythrocytes by high performance liquid chromatography. Rev Chim 66: 607-610, 2015.

10. Caruntu C, Boda D, Dumitrascu G, Constantin C and Neagu M: Proteomics focusing on immune markers in psoriatic arthritis. Biomark Med 9: 513-528, 2015. 
11. Batani A, Brănisteanu DE, Ilie MA, Boda D, Ianosi S, Ianosi G and Caruntu C: Assessment of dermal papillary and microvascular parameters in psoriasis vulgaris using in vivo reflectance confocal microscopy. Exp Ther Med 15: 1241-1246, 2018.

12. Boda CBD, Negrei C and Nicolescu F: Assessment of some oxidative stress parameters in methotrexate treated psoriasis patients. Farmacia 62: 704-710, 2014.

13. Kremer JM: Toward a better understanding of methotrexate. Arthritis Rheum 50: 1370-1382, 2004.

14. Newman M, Auerbach R, Feiner H, Holzman RS, Shupack J, Migdal P, Culubret M, Camuto P and Tobias H: The role of liver biopsies in psoriatic patients receiving long-term methotrexate treatment. Improvement in liver abnormalities after cessation of treatment. Arch Dermatol 125: 1218-1224, 1989.

15. Roenigk HH Jr, Auerbach R, Maibach H, Weinstein G and Lebwohl M: Methotrexate in psoriasis: Consensus conference. J Am Acad Dermatol 38: 478-485, 1998.

16. Hsu S, Papp KA, Lebwohl MG, Bagel J, Blauvelt A, Duffin KC, Crowley J, Eichenfield LF, Feldman SR, Fiorentino DF, et al; National Psoriasis Foundation Medical Board: Consensus guidelines for the management of plaque psoriasis. Arch Dermatol 148: 95-102, 2012.

17. Kalb RE, Strober B, Weinstein G and Lebwohl M: Methotrexate and psoriasis: 2009 National Psoriasis Foundation Consensus Conference. J Am Acad Dermatol 60: 824-837, 2009.

18. Summary of Product Characteristics: Annex I. https://www.ema. europa.eu/documents/product-information/nordimet-epar-productinformation_en.pdf.

19. Lim AYN, Gaffney K and Scott DGI: Methotrexate-induced pancytopenia: Serious and under-reported? Our experience of 25 cases in 5 years. Rheumatology (Oxford) 44: 1051-1055, 2005

20. Duhra P: Treatment of gastrointestinal symptoms associated with methotrexate therapy for psoriasis. J Am Acad Dermatol 28 466-469, 1993.

21. Temprano KK, Bandlamudi R and Moore TL: Antirheumatic drugs in pregnancy and lactation. Semin Arthritis Rheum 35: $112-121,2005$

22. Manna R, Verrecchia E, Diaco M, Montalto M, Cammarota G and Gasbarrini G: Folic acid supplementation during methotrexate treatment: Nonsense? Rheumatology (Oxford) 44 $563-564,2005$.
23. Murphy R: Folic acid reduces the efficacy of methotrexate. Nat Clin Pract Rheumatol 2: 4, 2006.

24. Whittle SL and Hughes RA: Folate supplementation and methotrexate treatment in rheumatoid arthritis: a review. Rheumatology (Oxford) 43: 267-271, 2004.

25. Lopez-Olivo MA, Siddhanamatha HR, Shea B, Tugwell P, Wells GA and Suarez-Almazor ME: Methotrexate for treating rheumatoid arthritis. Cochrane Database Syst Rev 10: CD000957, 2014.

26. Olteanu R, Zota A and Constantin M: Biosimilars: an update on clinical trials (review of published and ongoing studies). Acta Dermatovenerol Croat 25: 57-66, 2017.

27. Rodica O, Constantin MM,Zota A, Dorobantu DM, Constantin T, Serban ED, Balanescu P, Mihele D and Solovastru LG: Original clinical experience and approach to treatment study with interleukine 12/23 inhibitor in moderate-to-severe psoriasis patients. Farmacia 64: 918-921, 2016.

28. Tian $\mathrm{H}$ and Cronstein BN: Understanding the mechanisms of action of methotrexate: Implications for the treatment of rheumatoid arthritis. Bull NYU Hosp Jt Dis 65: 168-173, 2007.

29. Phillips DC, Woollard KJ and Griffiths HR: The anti-inflammatory actions of methotrexate are critically dependent upon the production of reactive oxygen species. Br J Pharmacol 138: 501-511, 2003.

30. Annest NM, VanBeek MJ, Arpey CJ and Whitaker DC: Intralesional methotrexate treatment for keratoacanthoma tumors: a retrospective study and review of the literature. J Am Acad Dermatol 56: 989-993, 2007.

31. Yoo MG and Kim IH: Intralesional methotrexate for the treatment of keratoacanthoma: Retrospective study and review of the korean literature. Ann Dermatol 26: 172-176, 2014

32. Goebeler M, Lurz C, Kolve-Goebeler ME and Bröcker EB: Pancytopenia after treatment of keratoacanthoma by single lesional methotrexate infiltration. Arch Dermatol 137: 1104-1105, 2001.

33. Cohen PR, Schulze KE and Nelson BR: Pancytopenia after a single intradermal infiltration of methotrexate. J Drugs Dermatol 4: 648-651, 2005. 